\title{
VÍTIMAS COM TRAUMATISMO CRANIOENCEFÁLICO NA SALA DE EMERGÊNCIA E FATOR ASSOCIADO À PERMANÊNCIA NO SETOR
}

\author{
VICTIMS WITH TRAUMATIC BRAIN INJURY IN THE \\ EMERGENCY ROOM AND FACTOR ASSOCIATED WITH \\ PERMANENCE IN THE SECTOR
}

\section{VÍCTIMAS CON TRAUMATISMO CRANEOENCEFÁLICO EN URGENCIAS Y FACTOR ASOCIADO A PERMANENCIA EN EL SECTOR}

\author{
Hosana da Silva ${ }^{1}$ \\ Lilia de Souza Nogueira ${ }^{2}$ \\ Regina Marcia Cardoso de Sousa ${ }^{3}$
}

Como citar este artigo: Silva H, Nogueira LS, Sousa RMC. Vítimas com traumatismo cranioencefálico na sala de emergência e fator associado à permanência no setor. Rev baiana enferm. 2021;25:e43056.

\begin{abstract}
Objetivo: analisar a evolução das vítimas de traumatismo cranioencefálico contuso na sala de emergência e identificar fatores independentes para tempo de permanência nesse serviço. Método: coorte prospectiva que incluiu todas as vítimas que atenderam aos critérios de elegibilidade e foram admitidas entre julho e dezembro de 2017 em hospital referência para trauma. Foi aplicado o Rapid Emergency Medicine Score para identificar a evolução das vítimas até 6 horas após admissão e aplicadas estatísticas descritivas e análise bivariada. Resultados: entre a admissão e 2 horas, foram observadas mudanças desfavoráveis em 35,1\% das vítimas, entre 2-4 horas em 13,6\% e entre 4-6 horas, em 42,8\%; foi observada melhora entre $27 \%$ e $28,6 \%$ da casuística. Suporte hemodinâmico foi fator independente para tempo de permanência. Conclusão: a evolução desfavorável foi mais frequente entre a admissão e 2 horas e após 4 horas. A maior permanência na sala de emergência ocorreu em vítimas com suporte hemodinâmico.
\end{abstract}

Descritores: Traumatismos Craniocerebrais. Traumatismos Cranianos Fechados. Serviço Hospitalar de Emergência. Enfermagem em Emergência. Resultado do Tratamento.

Objective: analyzing the evolution of victims of blunt traumatic brain injury in the emergency room and identifying independent factors for length of stay in this service. Method: a prospective cohort that included all victims who met the eligibility criteria and were admitted between July and December 2017 in a reference hospital for trauma. The Rapid Emergency Medicine Score was applied to identify the evolution of the victims up to 6 bours after admission and descriptive statistics and bivariate analysis were applied. Results: between admission and 2 bours, unfavorable changes were observed in $35.1 \%$ of the victims, between $2-4$ hours in $13.6 \%$ and between $4-6$ bours, in $42.8 \%$; improvement was observed between $27 \%$ and $28.6 \%$ of the sample. Hemodynamic support was an independent factor

Enfermeira. Mestre em Ciências. Enfermeira do Hospital das Clínicas da Faculdade de Medicina da Universidade de São Paulo. São Paulo, São Paulo, Brasil. http://orcid.org/0000-0002-1063-255X

Enfermeira. Doutora em Ciências. Professora Livre Docente do Departamento de Enfermagem Médico-Cirúrgica da Escola de Enfermagem da Universidade de São Paulo. São Paulo, São Paulo, Brasil. http://orcid.org/0000-000 I-5387-3807.

3 Enfermeira. Doutora em Ciências. Professora Titular do Departamento de Enfermagem Médico-Cirúrgica da Escola de Enfermagem da Universidade de São Paulo. São Paulo, São Paulo, Brasil. vian@usp.br. http://orcid.org/0000-0002-2575-7937. 
for length of stay. Conclusion: unfavorable evolution was more frequent between admission and 2 bours and after 4 hours. The longest stay in the emergency room occurred in victims with hemodynamic support.

Descriptors: Craniocerebral Trauma. Closed Head Trauma. Emergency Hospital Service. Emergency Nursing. Treatment Result.

Objetivo: analizar la evolución de las víctimas de traumatismo craneoencefálico contundente en la sala de urgencias e identificar factores independientes para la duración de la estancia en este servicio. Método: cohorte prospectiva que incluyó a todas las víctimas que cumplieron con los criterios de elegibilidad y estuvieron ingresadas entre julio y diciembre de 2017 en un hospital de referencia por traumatismo. Se aplicó el Rapid Emergency Medicine Score para identificar la evolución de las víctimas hasta 6 horas después del ingreso y se aplicó estadística descriptiva y análisis bivariado. Resultados: entre el ingreso y 2 horas, se observaron cambios desfavorables en 35, 1\% de las victimas, entre 2-4 horas en 13,6\% y entre 4-6 horas, en 42,8\%; se observó mejoría entre el 27\% y el 28,6\% de la muestra. El soporte hemodinámico fue un factor independiente para la duración de la estancia. Conclusión: la evolución desfavorable fue más frecuente entre el ingreso y 2 horas y después de 4 horas. La estancia más larga en la sala de urgencias ocurrió en víctimas con soporte bemodinámico.

Descriptores: Traumatismo Craneoencefálico. Traumatismo Craneoencefálico Cerrado. Servicio de Urgencias Hospitalarias. Enfermería de Emergencia. Resultado del Tratamiento.

\section{Introdução}

O traumatismo cranioencefálico (TCE) está entre as mais devastadoras lesões traumáticas e é uma das principais causas de morte e incapacidades persistentes no mundo, principalmente nas sociedades ocidentais ${ }^{(1-3)}$. No TCE, o dano total resultante do trauma é causado pela combinação do acometimento inicial e lesões secundárias, que resultam da progressão da lesão primária que ocorre durante o trauma. No entanto, lesões secundárias são potencialmente evitáveis ou tratáveis, o que torna o tratamento inicial das vítimas de grande importância em seu prognóstico ${ }^{(4-5)}$. Estudo realizado com 174 vítimas de TCE grave demonstrou que, nas primeiras 6 horas do trauma, a monitorização dos sinais vitais, como frequência cardíaca (FC) e pressão arterial média (PAM), juntamente com a pressão intracraniana, auxilia na identificação daqueles que requerem tratamento neurológico mais intensivo nos estágios iniciais após lesão encefálica ${ }^{(6)}$.

No Brasil, o TCE é responsável por mais de 125 mil admissões por ano e 9.700 mortes hospitalares nos últimos anos ${ }^{(7-8)}$. Os traumas envolvendo veículos são as causas mais comuns de TCE e a incidência dessa lesão está aumentando no mundo, especialmente nos países em desenvolvimento, em decorrência do uso crescente de transporte motorizado ${ }^{(3,7)}$.
O aumento das vítimas de TCE é de particular importância para os serviços de emergência, visto que, $80 \%$ a $92 \%$ dessas vítimas são tratadas nesse tipo de unidade ${ }^{(9)}$. Pesquisas que realizaram análises temporais indicaram crescimento dos atendimentos de vítimas de TCE em serviços de emergência, na medida em que encontraram taxas significativamente mais elevadas do que as anteriormente descritas na literatura ${ }^{(9)}$ ou compararam taxas relacionadas ao TCE de um longo período de acompanhamento ${ }^{(2,10)}$.

Em 2016, estudo americano mostrou que, entre 2001 e 2010, a quantidade de atendimentos em serviços de emergência por TCE aumentou $70 \%$ e o número de hospitalizações e mortes cresceu $11 \%$ e $7 \%$, respectivamente ${ }^{(10)}$. Investigação sobre a epidemiologia do TCE, publicada em 2020, enfatizou o constante aumento desses atendimentos, considerando dados de 2006 a 2014. Em 2014, os registros americanos documentaram 2,53 milhões de vítimas com TCE em serviços de emergência e aproximadamente 57 mil mortes relacionadas com essa lesão(2) . maior ônus do aumento dos casos de TCE recai em países de baixa ou média renda, onde, entre muitos outros problemas na área do atendimento à saúde, observa-se a superlotação dos serviços de emergência ${ }^{(11-14)}$. 
No Brasil, no ano de 2014, o Conselho Federal de Medicina (CFM) recomendou, na Resolução CFM no 2.077/14, máximo de 4 horas de permanência dos pacientes na sala de reanimação ou de procedimentos avançados, visando a melhoria dos desfechos ${ }^{(15)}$. Entretanto, não se pode descartar que os serviços de emergência encontram-se sob grande pressão em virtude da alta demanda de pacientes, falta de recursos disponíveis para atender as emergências e carência de leitos para a continuidade do tratamento, acarretando, assim, o aumento do tempo de permanência nesses serviços ${ }^{(11-14)}$. Esses problemas estruturais são largamente reconhecidos pelos gestores e profissionais de saúde, porém é provável que condições clínicas que dificultam a estabilização das vítimas de TCE também contribuam para ampliar a permanência desses pacientes na emergência.

Tendo em vista a importância do TCE entre as causas externas, os desdobramentos iniciais dessa lesão, os problemas da superlotação dos serviços de emergência e a ausência de investigações sobre a evolução de pacientes com TCE nesse setor, no Brasil e em estudos internacionais, questiona-se qual a evolução dessas vítimas na sala de emergência e quais os fatores associados ao aumento da permanência nesse setor?

A Organização Mundial da Saúde (OMS) tem reconhecido a escassez de boas evidências na literatura sobre o tratamento dos casos de TCE, especialmente nos países de baixa e média renda. Segundo a OMS, a falta de dados hospitalares sobre a assistência realizada a essas vítimas resulta em um progresso lento no atendimento das necessidades de saúde dessa população(3).

A frequência de vítimas de TCE nos serviços de emergência vem aumentando ${ }^{(2,9-10)}$ e estudos como este podem auxiliar na melhora das estratégias para tratar esses pacientes e ampliar o conhecimento da fisiopatologia do TCE nas primeiras horas após o evento traumático. Frente ao exposto, foram objetivos desta investigação: analisar a evolução das vítimas de TCE contuso durante a permanência na sala de emergência e identificar os fatores independentes para o tempo de permanência nesse serviço.

\section{Método}

Estudo de coorte prospectivo que analisou dados coletados na admissão do paciente, vítima de TCE contuso, na sala de emergência e 2, 4 e 6 horas após a admissão. Os participantes foram acompanhados, no máximo, até 6 horas na sala de emergência ou por menos tempo, isto é, até a saída do setor.

O estudo foi realizado no Serviço de Emergência de hospital universitário de grande porte localizado na cidade de São Paulo (SP), Brasil. É uma unidade de emergência referenciada para atendimento de vítimas de trauma, especializada em tratamento de neurocirurgia, que presta assistência a casos de alta complexidade.

Para este estudo foram incluídas todas as vítimas admitidas nessa unidade de emergência no período de julho a dezembro de 2017, com TCE contuso e idade maior ou igual a 15 anos, totalizando 79 pessoas. Foram excluídos os indivíduos que não apresentaram o TCE como lesão traumática principal, os admitidos no serviço de emergência após uma hora do evento traumático e aqueles que chegaram ao serviço em parada cardiorrespiratória e evoluíram a óbito, sem retorno à circulação espontânea após as manobras de reanimação.

Para identificar as vítimas que apresentaram TCE como lesão principal foi utilizada a Abbreviated Injury Scale (AIS), instrumento composto por uma lista de lesões apresentada no Manual AIS, que fornece, para cada descrição de lesão, um identificador composto por sete números. $\mathrm{O}$ último dígito desse identificador é o valor referente ao escore de gravidade da AIS, com pontuações que variam de um (menor gravidade) a seis pontos (máxima gravidade). A lista apresentada no manual AIS também classifica cada lesão decorrente do trauma por região corpórea considerando nove regiões: cabeça, face, pescoço, tórax, abdome, coluna vertebral, membros superiores, membros inferiores e superfície externa ${ }^{(16)}$.

Neste estudo, tendo em vista as regiões corpóreas e a gravidade das lesões, identificou-se a região que apresentou a lesão mais grave, isto é, com a mais alta pontuação na AIS. O TCE foi 
considerado lesão principal quando as vítimas tiveram a mais grave lesão na região da cabeça. Nos casos em que o mais alto escore esteve presente simultaneamente na cabeça e em outras regiões, prevaleceu a região com maior número de lesões, ao se estabelecer a localização da lesão principal.

$\mathrm{Na}$ análise da evolução das vítimas na sala de emergência, foi utilizado o Rapid Emergency Medicine Score (REMS) ${ }^{(17)}$, um indicador de gravidade fisiológico elaborado para ser utilizado em serviços de emergência, que inclui, para seu cálculo, a idade e os parâmetros fisiológicos: Escala de Coma de Glasgow (ECGl), FC, PAM, frequência respiratória (FR) e saturação periférica de oxigênio $\left(\mathrm{SpO}_{2}\right)$. Esses cinco componentes recebem valores de zero a +4 no cálculo do REMS e a pontuação atribuída à idade varia de zero a seis. A capacidade preditiva de mortalidade desse instrumento tem sido similar a outros índices que estimam a gravidade do trauma de pacientes em unidades de terapia intensiva e na emergência $^{(18)}$.

O REMS foi elaborado mediante regressão logística que analisou diversas características clínicas dos pacientes. Sua composição inclui os preditores independentes para mortalidade identificados em análise multivariada. Conforme proposto por esse instrumento, são atribuídas as seguintes pontuações aos pacientes: para menores de 45 anos, pontuação zero; para 45-54 anos, 2; para 55-64 anos, 3; para 65-74, 5; para maiores de 74 anos, 6. Em relação à PAM $(\mathrm{mmHg})$ os valores são: 70-109, escore zero; 110-129 ou 50-69, escore $2 ; 130-159$, escore $3 ;>159$ ou $\leq 49$, escore 4 . Quanto à FC (batimentos por minuto), os valores são: 70-109, escore zero; 110-139 ou 55-69, escore 2; 140-179 ou 40-54, escore 3; >179 ou $\leq 39$, escore 4. Para FR (movimentos respiratórios por minuto): 12-24, escore zero; 25-34 ou 10-11, escore 1; 6-9, escore 2; 35-49, escore 3; $>49$ ou $\leq 5$, escore 4 . Para $\mathrm{SpO}_{2}$ (\%): >89, escore zero; 86-89, escore 1; $75-85$, escore $3 ;<75$, escore 4 . Por fim, para ECGl: 14-15, escore zero; 11-13, escore 1; 8-10, escore 2; 5-7, escore 3 e $3-4$, escore $4^{(17)}$.

O REMS é obtido pela soma das pontuações atribuídas à idade e aos demais parâmetros. Seus valores variam de zero a 26. Escore inferior a 6 é indicativo de baixo risco de morrer; de 6 a 13 aponta risco intermediário; e superior a 13 , alto risco $^{(17)}$.

Nesta pesquisa, a evolução das vítimas foi analisada como variável categórica e considerada favorável, inalterada e desfavorável, tendo em vista as diferenças das pontuações do REMS e seus componentes fisiológicos entre a admissão e 2 horas, 2 e 4 horas e 4 e 6 horas após a chegada na sala de emergência. A evolução favorável foi identificada pelo resultado positivo na diferença de duas avaliações consecutivas do REMS; a inalterada, por diferença zero; e desfavorável, por diferença negativa. No REMS e seus componentes (ECGl, FC, PAM, FR, $\mathrm{SpO}_{2}$ e idade), as pontuações mais elevadas são indicativas de piores quadros clínicos; por isso, diferença positiva em duas avaliações consecutivas indicou melhora do paciente, e diferença negativa, piora.

Para identificar os fatores associados ao tempo de permanência na sala de emergência (variável dependente), foram analisadas as seguintes variáveis independentes: sexo, idade, REMS, ISS, ECGl, tipo de unidade utilizada no atendimento pré-hospitalar (APH) (básica ou avançada), suporte ventilatório, suporte hemodinâmico, sedação contínua e tratamento cirúrgico.

As informações de interesse para esta investigação foram coletadas e registradas em instrumento elaborado para este fim, por uma das pesquisadoras deste estudo, com auxílio de enfermeiras treinadas, que prestavam assistência aos participantes da pesquisa. Após a alta hospitalar, os prontuários dos pacientes selecionados para o estudo foram consultados, para identificar-se os diagnósticos das lesões e estimar a gravidade das vítimas segundo o Injury Severity Score (ISS) ${ }^{(16)}$.

O ISS indica a gravidade geral do traumatizado e é um dos índices de trauma mais utilizado mundialmente. Consideram-se, para determinar esse escore, todas as lesões diagnosticadas nas vítimas de trauma e as suas pontuações obtidas na AIS. O ISS é calculado pela soma do quadrado da AIS mais alta de três regiões corpóreas distintas. Para cálculo do ISS, as seis regiões corpóreas da AIS são agrupadas conforme segue: cabeça e pescoço; face; tórax; abdome e 
conteúdo pélvico; extremidades ou cintura pélvica; e superfície externa. O valor do ISS varia de 1 a 75 . O valor máximo pode ser resultado de três lesões em diferentes regiões corpóreas que receberam AIS 5 ou alguma pontuação 6. Para o cálculo do ISS, uma pontuação 6 na AIS representa sempre escore 75 , independentemente da gravidade ou presença de outras lesões.

Em relação à AIS, ao ISS e ao REMS, vale comentar que são indicadores utilizados mundialmente para identificar a gravidade das lesões traumáticas, do trauma e dos pacientes na sala de emergência, respectivamente. Além disso, a conformação e a objetividade de seus componentes (diagnósticos de lesões, idade e parâmetros fisiológicos) têm dispensado a adaptação transcultural desses instrumentos para uso em diferentes países.

As informações coletadas para esta pesquisa foram armazenadas em banco de dados com uso do software Microsoft Office Excel 2007 e para a análise aplicou-se o software Statistical Package for the Social Sciences (SPSS) versão 22. A consistência do banco de dados foi verificada utilizando-se o programa Excel, por meio de filtros, tabelas unidimensionais e dinâmicas.

Estatísticas descritivas foram usadas para caracterização da amostra em relação às variáveis de interesse e para analisar a evolução das vítimas na sala de emergência. As variáveis categóricas foram analisadas por meio de frequências absolutas e relativas; para as numéricas foram calculados a média e o desvio-padrão. Para identificar os fatores associados ao tempo de permanência na sala de emergência, aplicou-se o Coeficiente de Correlação de Person, quando a análise foi realizada entre duas variáveis de escala métrica. O Teste $\mathrm{T}$ de Student foi utilizado para comparar as médias de permanência na sala de emergência de dois grupos independentes, quando verificada distribuição normal dos dados. Nos casos de distribuição não normal, foi aplicado o teste não paramétrico de Wilcoxon-Mann-Whitney na comparação dos tempos de permanência. Para testar a aderência à normalidade, aplicou-se o Teste de Shapiro-Wilk. Para as análises inferenciais, considerou-se o nível de significância de 5\%.
Este estudo foi aprovado pelo Comitê de Ética em Pesquisa (CEP) em 18/5/2017, Certificado de Apresentação de Apreciação Ética (CAAE), $\mathrm{n}$ ㅇ 65147317.0.0000.5392. O Termo de Consentimento Livre e Esclarecido (TCLE) e o Termo de Assentimento Livre e Esclarecido (TALE) foram aplicados, conforme pertinência, para certificar a adesão dos participantes à pesquisa.

\section{Resultados}

Das 79 vítimas com idade maior ou igual a 15 anos e TCE contuso, admitidas na sala de emergência no período de coleta de dados, 4 chegaram ao serviço em parada cardiorrespiratória, sem sucesso nas tentativas de reanimação. Foram admitidos no hospital após uma hora do evento traumático 20 pacientes, 7 não apresentaram TCE como lesão principal e 2 recusaram-se a participar do estudo. Restaram, portanto, 46 pacientes com TCE contuso que compuseram a casuística desta pesquisa.

A maioria dessas vítimas (63\%) esteve envolvida em acidentes de trânsito ou quedas (28,3\%), era do sexo masculino (84,7\%), com idade média de 34,7 anos $( \pm 15,1)$. Na admissão na sala de emergência, o valor médio do REMS foi de 4,0 $( \pm 2,5)$ e a variação de zero a 11 . Em relação à gravidade do trauma, a média do ISS foi de 11,8 $( \pm 7,7)$ e a maioria $(54,4 \%)$ teve indicação de TCE grave pela ECGl (média de $8,3 \pm 5,0$ ).

Foram socorridos por serviço de APH móvel $93,5 \%$ das vítimas e $6,5 \%$ não tiveram esse atendimento. Equipes de suporte básico de vida e suporte avançado de vida atenderam, respectivamente, $52,2 \%$ e $41,3 \%$ da casuística.

As intervenções relacionadas com suporte ventilatório foram as mais frequentes na sala de emergência, utilizadas em 87,0\% dos casos. Verificou-se que a maioria das vítimas, 65,0\%, esteve com intubação orotraqueal na sala de emergência. Cateter e máscara de oxigênio foram utilizados em 22,0\% dos casos. A administração de volume ocorreu em 18 vítimas, sendo em 15 pacientes como conduta isolada e em 3 acompanhada de drogas vasoativas, 2 dos quais também utilizaram hemocomponentes. Uma vítima recebeu somente droga vasoativa na 
sala de emergência, totalizando 19 participantes com suporte hemodinâmico.

Receberam sedação contínua na sala de emergência 16 pacientes, 34,8\% da casuística. Cerca de um terço das vítimas, 32,6\%, tiveram tratamento cirúrgico e foram encaminhadas diretamente da sala de emergência para o centro cirúrgico.

A maior parte $(34,7 \%)$ dos participantes deste estudo ainda ficou em observação no pronto-socorro depois da saída da sala de emergência. Para o centro cirúrgico foram encaminhados 32,6\% dos pacientes e 21,7\% para a unidade de cuidados intensivos. Dois $(4,4 \%)$ pacientes evoluíram a óbito na sala de emergência, dois $(4,4 \%)$ foram encaminhados para a unidade de internação e um (2,2\%) foi transferido para outro hospital.

$\mathrm{Na}$ Tabela 1, observa-se que as evoluções desfavoráveis, segundo o REMS, ocorreram em 35,1\% das vítimas nas duas primeiras horas após admissão na sala de emergência. A maior frequência de evolução desfavorável ocorreu no intervalo de 4 a 6 horas em 42,8\% dos casos que permaneceram na sala de emergência por esse tempo. O percentual de pacientes que evoluíram com melhora foi similar nos três intervalos de tempo, de $27 \%$ a $28,6 \%$.

Tabela 1 - Vítimas de traumatismo cranioencefálico contuso segundo evolução pelo Rapid Emergency Medicine Score nos intervalos de avaliação. São Paulo, São Paulo, Brasil - 2017. (N=37)

\begin{tabular}{l|c|c|c|c|c|c}
\hline \multirow{2}{*}{$\begin{array}{l}\text { Evolução pelo Rapid Emergency Medicine } \\
\text { Score }\end{array}$} & \multicolumn{4}{c}{ Intervalos de avaliação } \\
\cline { 2 - 7 } & \multicolumn{2}{c}{$\begin{array}{c}\text { Admissão até } \mathbf{2} \\
\text { horas }\end{array}$} & \multicolumn{2}{c}{$\mathbf{2}$ a 4 horas } & \multicolumn{2}{|c}{$\mathbf{4}$ a $\mathbf{6}$ horas } \\
\cline { 2 - 7 } & $\mathbf{n}$ & $\mathbf{0}$ & $\mathbf{n}$ & $\mathbf{\%}$ & $\mathbf{n}$ & $\mathbf{\%}$ \\
\hline Favorável (1) & 10 & 27,0 & 6 & 27,3 & 4 & 28,6 \\
Inalterada (2) & 14 & 37,9 & 13 & 59,1 & 4 & 28,6 \\
Desfavorável (3) & 13 & 35,1 & 3 & 13,6 & 6 & 42,8 \\
Total & 37 & 100,0 & 22 & 100,0 & 14 & 100,0 \\
\hline
\end{tabular}

Fonte: Elaboração própria.

(1) Diferença positiva entre avaliações consecutivas pelo REMS.

(2) Diferença zero entre avaliações consecutivas pelo REMS.

(3) Diferença negativa entre avaliações consecutivas pelo REMS.

O tempo de permanência dos pacientes na sala de emergência variou de 30 a 1.080 minutos (18 horas). Em média, a permanência foi de 295,3 $( \pm 274,5)$ minutos, cerca de 4 horas e meia. Nove participantes permaneceram na sala de emergência por menos de 2 horas, 15 vítimas foram transferidas entre 2 e 4 horas e 8 no intervalo de 4 a 6 horas. Por consequência, observa-se, nas Tabelas 1 e 2, que as avaliações duas horas após admissão na emergência tiveram 37 participantes; nas realizadas quatro horas após, participaram 22 vítimas, das quais 14 (30,3\%) permaneceram na sala de emergência por tempo de 6 horas ou mais.

Tabela 2 - Vítimas de traumatismo cranioencefálico contuso segundo evolução nos parâmetros fisiológicos do Rapid Emergency Medicine Score nos intervalos de avaliação. São Paulo, São Paulo, Brasil - 2017

(continua)

\begin{tabular}{l|c|c|c|c|c|c}
\hline \multirow{2}{*}{ Parâmetros } & \multicolumn{3}{|c}{ Evolução - entre admissão e 2 horas após (N=37) } \\
\cline { 2 - 7 } & \multicolumn{2}{|c|}{ Favorável (1) } & \multicolumn{2}{c}{ Inalterada (2) } & \multicolumn{2}{c}{ Desfavorável (3) } \\
\cline { 2 - 7 } & $\mathbf{n}$ & $\mathbf{0}$ & $\mathbf{n}$ & $\mathbf{0}$ & $\mathbf{n}$ & $\mathbf{0}$ \\
\hline Escala de Coma de Glasgow & 2 & 5,4 & 28 & 75,7 & 7 & 18,9 \\
Frequência cardíaca & 6 & 16,2 & 24 & 64,9 & 7 & 18,9 \\
Pressão arterial média & 6 & 16,2 & 27 & 73,0 & 4 & 10,8 \\
Frequência respiratória & 3 & 8,1 & 27 & 73,0 & 7 & 18,9 \\
Saturação periférica de oxigênio & - & - & 35 & 94,6 & 2 & 5,4
\end{tabular}


Tabela 2 - Vítimas de traumatismo cranioencefálico contuso segundo evolução nos parâmetros fisiológicos do Rapid Emergency Medicine Score nos intervalos de avaliação. São Paulo, São Paulo, Brasil - 2017

(conclusão)

\begin{tabular}{|c|c|c|c|c|c|c|}
\hline \multirow{3}{*}{ Parâmetros } & \multicolumn{6}{|c|}{ Evolução - entre 2 e 4 horas após admissão (N=22) } \\
\hline & \multicolumn{2}{|c|}{ Favorável (1) } & \multicolumn{2}{|c|}{ Inalterada (2) } & \multicolumn{2}{|c|}{ Desfavorável (3) } \\
\hline & $\mathbf{n}$ & $\%$ & $\mathbf{n}$ & $\%$ & $\mathbf{n}$ & $\%$ \\
\hline Escala de Coma de Glasgow & - & - & 22 & 100,0 & - & - \\
\hline Frequência cardíaca & 5 & 22,7 & 12 & 54,6 & 5 & 22,7 \\
\hline Pressão arterial média & 1 & 4,5 & 19 & 86,4 & 2 & 9,1 \\
\hline Frequência respiratória & 3 & 13,6 & 19 & 86,4 & - & - \\
\hline \multirow[t]{2}{*}{ Saturação periférica de oxigênio } & - & - & 22 & 100,0 & - & - \\
\hline & \multicolumn{6}{|c|}{ Evolução - entre 4 e 6 horas após admissão (N=14) } \\
\hline \multirow[t]{2}{*}{ Parâmetros } & \multicolumn{2}{|c|}{ Favorável (1) } & \multicolumn{2}{|c|}{ Inalterada (2) } & \multicolumn{2}{|c|}{ Desfavorável (3) } \\
\hline & $\mathbf{n}$ & $\%$ & $\mathbf{n}$ & $\%$ & $\mathbf{n}$ & $\%$ \\
\hline Escala de Coma de Glasgow & - & - & 14 & 100,0 & - & - \\
\hline Frequência cardíaca & 2 & 14,3 & 9 & 64,3 & 3 & 21,4 \\
\hline Pressão arterial média & 3 & 21,4 & 8 & 57,2 & 3 & 21,4 \\
\hline Frequência respiratória & - & - & 13 & 92,9 & 1 & 7,1 \\
\hline Saturação periférica de oxigênio & - & - & 14 & 100,0 & _- & - \\
\hline
\end{tabular}

Fonte: Elaboração própria.

Nota: Sinal convencional utilizado:

- Dado numérico igual a zero não resultante de arredondamento.

(1) Diferença positiva entre avaliações consecutivas considerando as pontuações atribuídas aos parâmetros pelo REMS.

(2) Diferença zero entre avaliações consecutivas considerando as pontuações atribuídas aos parâmetros pelo REMS.

(3) Diferença negativa entre avaliações consecutivas considerando as pontuações atribuídas aos parâmetros pelo REMS.

Conforme se observa na Tabela 2, nas duas primeiras horas na sala de emergência, foram observados casos com evolução desfavorável em relação a todos os parâmetros. A FC, a ECGl e a FR apresentaram maior percentual de vítimas com essa evolução, 18,9\% cada. A $\mathrm{SpO}_{2}$ foi o parâmetro mais estável em todo o período de avaliação e somente dois pacientes tiveram alteração desfavorável, que ocorreu nas duas primeiras horas após admissão na sala de emergência. A ECGl, que apresentou uma das maiores frequências de evolução desfavorável inicialmente, manteve-se estável nos pacientes que permaneceram na sala de emergência após 2 horas de sua admissão. Nas avaliações após 2 horas, a FC e a PAM foram os únicos parâmetros que apresentaram frequências expressivas de alterações desfavoráveis.

Tabela 3 - Testes de associação entre tempo de permanência na sala de emergência e as variáveis sexo, idade, gravidade e características do atendimento pré e intra-hospitalar. São Paulo, São Paulo, Brasil - 2017. $(\mathrm{N}=46)$

(continua)

\begin{tabular}{|c|c|c|}
\hline Variáveis & $\begin{array}{c}\text { Coeficiente de } \\
\text { correlação }\end{array}$ & p-valor \\
\hline Sexo e permanência & & $0,596(2)$ \\
\hline Idade e permanência & $-0,155$ & $0,303(3)$ \\
\hline Rapid Emergency Medicine Score na admissão e permanência & 0,111 & $0,461(3)$ \\
\hline Injury Severity Score e permanência & 0,137 & $0,366(3)$ \\
\hline Escala de Coma de Glasgow e permanência & $-0,174$ & $0,247(3)$ \\
\hline Unidade utilizada no Atendimento Pré-hospitalar (1) e & & $0,200(4)$ \\
\hline
\end{tabular}

permanência 
Tabela 3 - Testes de associação entre tempo de permanência na sala de emergência e as variáveis sexo, idade, gravidade e características do atendimento pré e intra-hospitalar. São Paulo, São Paulo,

Brasil - 2017. (N=46)

(conclusão)

\begin{tabular}{|c|c|c|}
\hline Variáveis & $\begin{array}{l}\text { Coeficiente de } \\
\text { correlação }\end{array}$ & p-valor \\
\hline Suporte ventilatório e permanência & & $0,062(4)$ \\
\hline Suporte hemodinâmico e permanência & & $0,016(4)$ \\
\hline Sedação contínua e permanência & & $0,252(4)$ \\
\hline Tratamento cirúrgico e permanência & & $0,505(4)$ \\
\hline
\end{tabular}

Fonte: Elaboração própria.

(1) Vítimas sem atendimento pré-hospitalar foram excluídas.

(2) Teste de Wilcoxon-Mann-Whitney.

(3) Teste de Correlação de Person.

(4) Teste T de Student.

Conforme se observa na Tabela 3, ocorreu diferença estatisticamente significativa no tempo de permanência na sala de emergência somente em relação ao uso de suporte hemodinâmico nesse serviço ( $\mathrm{p}$ valor=0,016). Vítimas que necessitaram de droga vasoativa, reposição de volume e/ou hemocomponentes permaneceram na sala de emergência por maior tempo.

\section{Discussão}

A finalidade do serviço de emergência é avaliar, estabilizar, diagnosticar e encaminhar a vítima ao local adequado para seu tratamento definitivo o mais breve possível; é um setor onde o paciente deve permanecer por pouco tempo. Modelos de atendimentos de emergência de vários países têm como alvo o tempo de permanência máxima de 4 horas nos serviços de emergência $^{(12)}$, porém estudos que analisam pacientes com longo tempo de permanência nesse setor consideram cortes de tempo de 2 a 6 horas nas análises de suas pesquisas ${ }^{(19-22)}$.

Neste estudo, foi expressiva a frequência de pacientes da casuística que estiveram 6 ou mais horas na sala de emergência (30,3\%) e, entre eles, a evolução desfavorável alcançou o seu maior patamar (42,8\% dos casos). Esse resultado corrobora a recomendação de, no máximo, 4 horas de permanência dentro da sala de emergência e com as indicações que apontam o período $<6$ horas como tempo limite para permanência nesse setor ${ }^{(22)}$.
Vale comentar que, nas primeiras 4 horas na sala de emergência, observou-se evolução desfavorável mais frequentemente nas 2 horas iniciais após admissão na unidade. Essas primeiras horas após admissão no serviço de emergência têm sido reconhecidas como o período direcionado para avaliação da vítima, definição diagnóstica e início do plano de tratamento ${ }^{(12)}$, o que pode explicar os resultados observados.

Especialmente em pacientes com TCE, a presença de hipóxia somada à hipotensão está associada a um aumento de risco de morte e piores desfechos; portanto, todo o esforço da equipe multidisciplinar que atende essas vítimas é voltado para a precoce estabilização hemodinâmica e ventilatória na sala de emergência ${ }^{(23)}$. Em relação à evolução dos componentes do REMS, a ECGl e os parâmetros respiratórios (FR e $\mathrm{SpO}_{2}$ ) permaneceram estáveis duas horas após a admissão nesse setor. As intervenções de suporte ventilatório foram as mais utilizadas (87,0\% dos casos) e, provavelmente, foram essenciais para a rápida estabilização da $\mathrm{FR}$ e $\mathrm{SpO}_{2}$ das vítimas.

A ECGl é um dado importante na avaliação da evolução de lesão cerebral aguda. A estabilidade desse parâmetro após duas horas na sala de emergência é um indicador de que as vítimas de TCE da presente investigação beneficiaram-se com as intervenções recebidas. Em geral, nas horas e dias subsequentes ao TCE, um conjunto de lesões secundárias, como isquemia celular, ativação da cascata inflamatória, edema 
dos neurônios e astrócitos e edema vasogênico, exacerba o dano inicial. Dentro dos rígidos limites do crânio, o edema aumenta a pressão intracraniana e reduz a pressão de perfusão cerebral. O agravamento da isquemia cerebral, com a redução da pressão de perfusão cerebral e o aumento do edema e da hipertensão intracraniana, contribui para um ciclo vicioso de piora do quadro clínico da vítima ${ }^{(24)}$.

Neste estudo, a sedação contínua, essencial para diminuir o metabolismo e o consumo de oxigênio cerebral e proteger o encéfalo de danos decorrentes da lesão ${ }^{(24)}$, foi utilizada em 34,8\% da casuística e pode ter prevenido e reduzido as lesões secundárias, ocasionando a precoce estabilidade da ECGl das vítimas. No entanto, há de se considerar que o paciente sedado apresenta depressão do nível de consciência induzida por fármacos e que a sedação prejudica a avaliação da função encefálica. Valores estáveis da ECGl, após duas horas da admissão, podem ser parcialmente decorrentes da sedação contínua, que inicialmente rebaixa a responsividade do paciente aos estímulos externos, mas, na sequência, mantém a ECGl em valores estáveis.

Em todos os períodos de avaliação, as alterações hemodinâmicas desfavoráveis, detectadas pela FC e PAM, ocorreram em frequência importante. Há diferentes motivos para acontecerem alterações hemodinâmicas na vítima de TCE, entre eles, as lesões cerebrais, que podem afetar diretamente as funções cardiovasculares, e as perdas sanguíneas, importantes em decorrência de lesões associadas. Por outro lado, a hipertensão arterial observada na tríade de Cushing é encontrada nos casos graves de aumento da pressão intracraniana e pode ser lesiva ao paciente por aumentar o edema vasogênico resultante do trauma ${ }^{(23-24)}$.

Em relação aos resultados relacionados às alterações hemodinâmicas, deve-se também ponderar que as vítimas apresentaram o TCE como lesão principal. Portanto, houve menor possibilidade de grandes perdas sanguíneas, devido às características desse tipo de ferimento. No entanto, a presença de lesões extracranianas importantes pode ter desencadeado sangramentos e quedas significativas da pressão arterial. Em contrapartida, o tratamento inicial do TCE concentra-se na prevenção de lesões secundárias, por meio de condutas precoces para evitar a hipotensão e a hipóxia, visando a manutenção da pressão de perfusão cerebral adequada e, por extensão, do fluxo sanguíneo cerebral ${ }^{(25)}$.

Em relação à investigação dos fatores associados ao tempo de permanência na sala de emergência das vítimas deste estudo, as análises indicaram que não houve relação entre as variáveis sexo, idade, gravidade, características do APH e duração da estada no serviço. Estudos anteriores identificaram grupos de pacientes que são propensos a permanecer mais do que 4 ou 6 horas no serviço de emergência. Seus resultados apontaram que pacientes mais velhos (65 anos ou mais), do sexo feminino, com queixas de maior complexidade, que chegaram ao serviço em horário de pico dos atendimentos ou no período noturno, que necessitaram de intervenções cirúrgicas, atendimento clínico ou neurológico, precisaram de exames radiológicos ou laboratoriais, entre outras razões, permaneceram por tempo mais prolongado nesse setor ${ }^{(20-21)}$.

$\mathrm{Na}$ comparação com o presente estudo, diferentes variáveis foram analisadas, assim como foram observados resultados diversos em relação ao sexo e à idade. Indubitavelmente, as especificidades das vítimas de trauma, jovens, na maioria do sexo masculino, descritas na literatura ${ }^{(2,19,21)}$ e também constatadas neste estudo (idade média de $34,7 \pm 15,2$ anos e $84,7 \%$ da casuística do sexo masculino), estiveram relacionadas com esses resultados.

Nesta pesquisa, o ISS não teve correlação com tempo de permanência no serviço de emergência. Igual resultado foi encontrado em investigação que analisou traumatizados não cirúrgicos graves que necessitavam de terapia intensiva ${ }^{(19)}$. Diferentemente desses resultados, análise de vítimas de trauma estáveis hemodinamicamente (pressão arterial sistólica >100 $\mathrm{mmHg}, \mathrm{FC}<110$ batimentos/min) mostrou que o escore desse índice foi significativamente maior no grupo com prolongado tempo de permanência na emergência ${ }^{(21)}$. Provavelmente, a estabilidade hemodinâmica dos pacientes dessa investigação favoreceu para que a gravidade do 
trauma, estabelecida pelo ISS, se associasse com a permanência nesse setor.

O suporte hemodinâmico foi a única variável deste estudo que se associou à maior permanência das vítimas de TCE na sala de emergência. Embora 41,3\% da casuística tenha recebido esse tratamento, alterações de FC e PAM foram expressivas em todos os períodos de avaliação. Pelos resultados, a estabilidade hemodinâmica foi a meta mais difícil de alcançar na sala de emergência e as alterações da PAM e FC foram responsáveis pela piora dos valores do REMS da quase totalidade dos casos após 2 horas da admissão.

Como implicações práticas dos achados desta pesquisa, pode-se salientar que os resultados reforçam a recomendação da Resolução CFM $\mathrm{n}^{\mathrm{o}} 2.077 / 14^{(15)}$ de permanência máxima na sala de emergência de até 4 horas após admissão. Na última década, várias intervenções para qualificar a assistência no serviço de emergência têm sido propostas, entre elas destacam-se as regras de tempo máximo de permanência no setor, a exemplo da "Regra de 4 horas" no Reino Unido ${ }^{(22)}$ e a própria Resolução CFM $n^{\circ}$ $2.077 / 14^{(15)}$. É explicitado, nas pesquisas, que o menor tempo de permanência tem sido considerado como elemento-chave na qualidade do serviço de emergência ${ }^{(12,20-22)}$.

Os resultados também ressaltaram os intervalos de tempo com as maiores frequências de evolução desfavorável das vítimas (nas primeiras 2 horas após admissão na sala de emergência e entre 4 e 6 horas após), evidenciando períodos que os pacientes necessitavam de redobrada monitorização da equipe de enfermagem e de estratégias assistenciais e de gestão do enfermeiro que evitassem sua piora clínica. Além disso, os achados indicam especial atenção às vítimas com TCE e suporte hemodinâmico, visto que permaneciam por mais tempo na sala de emergência e apresentavam processo mais moroso de estabilização clínica.

Estudo que analisou as causas básicas de longo tempo de permanência na emergência (> 6 horas) identificou que, aproximadamente, $76 \%$ dessas causas eram de caráter organizacional e $22 \%$ relacionadas ao paciente ou à doença ${ }^{(20)}$. Entre os fatores relacionados ao caráter organizacional, a superlotação dos serviços de emergência tem se destacado, uma vez que se associa com resultados adversos aos pacientes, incluindo aumento da mortalidade ${ }^{(20)}$. O fator mais comumente relatado como responsável pela superlotação do setor de emergência é a falta de leitos hospitalares. De mais a mais, a superlotação afeta o acesso oportuno aos cuidados necessários ao paciente, acarretando atrasos no atendimento, quase sempre prejudiciais para aqueles que recebem e que cuidam ${ }^{(22)}$.

É importante ressaltar, como limitações deste estudo, que a investigação se restringiu a descrever a evolução das vítimas na sala de emergência sem abordar seu impacto nos resultados do TCE a médio e longo prazo. Ademais, a realização do estudo em um único local, centro de referência com recursos avançados para a população-alvo desta pesquisa, pode ter propiciado uma evolução mais favorável das vítimas na sala de emergência do que em outros serviços.

Outra limitação refere-se ao objetivo desta investigação, conduzido para identificar características clínicas para longa permanência no serviço de emergência, sem considerar a interferência de aspectos organizacionais.

\section{Conclusão}

A análise da evolução das vítimas de TCE contuso nas primeiras 6 horas após admissão na sala de emergência evidenciou mudanças expressivas nas condições fisiológicas dos pacientes. A melhora de mais de $25 \%$ da casuística foi observada em todas as avaliações realizadas até 6 horas. Entre a admissão na sala de emergência e 2 horas após, a evolução desfavorável $(35,1 \%)$ foi mais frequente do que favorável (27\%) e alcançou seu ápice entre 4 e 6 horas (42,8\%). A estabilidade da ECGl e $\mathrm{SpO}_{2}$ foi identificada até duas horas após admissão no serviço. Por outro lado, as alterações hemodinâmicas desfavoráveis, detectadas pela FC e PAM, ocorreram em frequência importante em todas as avaliações. 
O uso do suporte hemodinâmico foi fator independente para o tempo de permanência na sala de emergência. Vítimas que receberam esse tratamento permaneceram mais tempo nesse setor.

\section{Fontes de Financiamento:}

Coordenação de Aperfeiçoamento de Pessoal de Nível Superior - Brasil (Capes) - Código de Financiamento 001.

\section{Colaborações:}

1 - concepção, projeto, análise e interpretação dos dados: Hosana da Silva e Regina Marcia Cardoso de Souza;

2 - redação do artigo e revisão crítica relevante do conteúdo intelectual: Hosana da Silva, Lilia de Souza Nogueira e Regina Marcia Cardoso de Sousa;

3 - aprovação final da versão a ser publicada: Hosana da Silva, Lilia de Souza Nogueira e Regina Marcia Cardoso de Sousa.

\section{Referências}

1. Maas AIR, Menon DK, Adelson PD, Andelic N, Bell MJ, Belli A, et al. Traumatic brain injury: integrated approaches to improve prevention, clinical care, and research. Lancet Neurol. 2017;16(12):987-1048. DOI: https://doi.org/10. 1016/S1474-4422(17)30371-X

2. Capazzi A, WooJ, Verduzco-Gutierrez M. Traumatic Brain Injury: An Overview of Epidemiology, Pathophysiology, and Medical Management. Med Clin North Am. 2020;104(2):213-38. DOI: https:// doi.org/10.1016/j.mcna.2019.11.001

3. Mehmood A, Zia N, Kobusingye O, Namaganda RH, Ssenyonjo H, Kiryabwire J, et al. Determinants of emergency department disposition of patients with traumatic brain injury in Uganda: results from a registry. Trauma Surg Acute Care Open. 2018;3(1):e000253. DOI: https://doi.org/10.1136/ tsaco-2018-000253

4. Dash HH, Chavali S. Management of traumatic brain injury patients. Korean J Anesthesiol. 2018;71(1):12-21. DOI: https://doi.org/10.4097/ kjae.2018.71.1.12
5. Geeraerts T, Velly L, Abdennour L, Asehnoune K, Audibert G, Bouzat P, et al. Management of severe traumatic brain injury (first 24 hours). Anaesth Crit Care Pain Med. 2018;37(2):171-86. DOI: https:// doi.org/10.1016/j.accpm.2017.12.001

6. Gao L, Smielewski P, Czosnyka M, Ercole A. Cerebrovascular Signal Complexity Six Hours after Intensive Care Unit Admission Correlates with Outcome after Severe Traumatic Brain Injury. J Neurotrauma. 2016;33(22):2011-8. DOI: http:// doi.org/10.1089/neu.2015.4228

7. Almeida CER, Sousa Filho JL, Dourado JC, Gontijo PAM, Dellaretti MGMA, Costa BS. Traumatic brain injury epidemiology in Brazil. World Neurosurg. 2016;87:540-7. DOI: https://doi. org/10.1016/j.wneu.2015.10.020

8. Brasil. Ministério da Saúde. Informações de Saúde (TABNET). Epidemiológicas e Morbidade 2008-2020 [Internet]. Brasília (DF); 2020 [cited 2021 Jan 9]. Available from: http://www2.datasus.gov. br/DATASUS/index.php?area $=0203 \& i d=6926$

9. Fu TS, Jing R, Fu WW, Cusimano MD. Epidemiological Trends of Traumatic Brain Injury Identified in the Emergency Department in a Publicly-Insured Population, 2002-2010. PLoS One. 2016;11(1):e0145469. DOI: https://doi. org/10.1371/journal.pone.0145469

10. Taylor CA, Bell MJ, Breiding MJ, Xu L. Traumatic Brain Injury-Related Emergency Department Visits, Hospitalizations, and Deaths - United States, 2007 and 2013. MMWR Surveill Summ. 2017;66(9):1-16. DOI: http://dx.doi. org/10.15585/mmwr.ss6609a1

11. Salway RJ, Valenzuela R, Shoenberger JM, Mallon WK, Viccellio A. Emergency Department (ED) Overcrowding: Evidence-Based Answers To Frequently Asked Questions. Rev Méd Clín Condes. 2017;28(2):213-9. DOI: https://doi.org/10.1016/j. rmclc.2017.04.008

12. Oliveira AC. Modelos de organização de serviços de urgência: uma revisão da literatura [Internet]. Brasília (DF): Programa de Apoio ao Desenvolvimento Institucional do Sistema Único de Saúde; 2014 [cited 2021 Jan 9]. Available from: http://portalarquivos2.saude.gov.br/images/ pdf/2016/setembro/26/7-Revisao-Urg--ncias.pdf

13. Vermeulen MJ, Guttmann A, Stukel TA, Kachra A, Sivilotti MLA, Rowe BH, et al. Are reductions in emergency department length of stay associated with improvements in quality 
of care? A difference-in-differences analysis. BMJ Qual Saf. 2016;25(7):489-98. DOI: http://dx.doi. org/10.1136/bmjqs-2015-004189

14. van der Veen D, Remeijer C, Fogteloo AJ, Heringhaus C, Groot B. Independent determinants of prolonged emergency department length of stay in a tertiary care centre: a prospective cohort study. Scand J Trauma Resusc Emerg Med. 2018;26(1):81. DOI: https://doi.org/10.1186/ s13049-018-0547-5

15. Conselho Federal de Medicina. Resolução CFM 2077/14. Dispõe sobre a normatização do funcionamento dos serviços hospitalares de urgência e emergência, dimensionamento da equipe médica e do sistema de trabalho [Internet]. Brasília (DF); 2014 [cited 2021 Jan 9]. Available from: https://portal.cfm.org.br/images/ PDF/resolucao2077.pdf

16. Association for the Advancement of Automotive Medicine. The Abbreviated Injury Scale (AIS): 2015 Revision. Des Plaines, Illinois (US); 2016.

17. Olsson T, Terent A, Lind L. Rapid Emergency Medicine score: a new prognostic tool for in-hospital mortality nonsurgical emergency department patients. J Intern Med. 2004;255(5): 579-87. DOI: https://doi.org/10.1111/j.1365-2796. 2004.01321.x

18. Lima KP, Nogueira LS, Barbosa G, Bomfim AKS, Sousa RMC. Severity indexes of blunt trauma victims in intensive therapy: prediction capacity for mortality. Rev Esc Enferm USP. 2021;31(55):e3747. DOI: https://doi.org/10.1590/ S1980-220X2020003203747

19. Siletz A, Jin K, Cohen M, Lewis C, Tillou A, Cryer HM, et al. Emergency department length of stay in critical nonoperative trauma. J Surg
Res. 2017;214:102-8. DOI: http://dx.doi.org/10. 1016/j.jss.2017.02.079

20. Driesen BEJM, van Riet BHG, Verkerk L, Bonjer HJ, Merten H, Nanayakkara PWB. Long length of stay at the emergency department is mostly cause by organisational factors outside the influence of the emergency department: A root cause analysis. PLoS One. 2018;13(9):e0202751. DOI: http://dx.doi.org/10.1371/journal.pone. 0202751

21. Becker A, Segal G, Berlin Y, Hershko D. The emergency department length of stay: Is the time running out? Clin J Traumatol. 2019;22(3):125-8. DOI: http://dx.doi.org/10.1016/j. cjtee.2019.01.008

22. Hosseininejad SM, Aminiahidashti H, Pashaei SM, Khatir IG, Montazer SH, Bozorgi F, et al. Determinants of Prolonged Length of Stay in the Emergency Department; a Cross-sectional Study. Emerg (Tehran) [Internet]. 2017 [cited 2021 May 18];5(1):e53. Available from: https://www.ncbi. nlm.nih.gov/pmc/articles/PMC5325925/

23. American College of Surgeons. ATLS Advanced Trauma Life Support (Student Course Manual). 10a ed. Chicago (IL); 2018.

24. Ng SY, Lee AYW. Traumatic Brain Injuries: Pathophysiology and Potential Therapeutic Targets. Front Cell Neurosci. 2019;13:528. DOI: https://doi.org/10.3389/fncel.2019.00528

25. Vella MA, Crandall ML, Patel MB. Acute Management of Traumatic Brain Injury. Surg Clin North Am. 2017;97(5):1015-30. DOI: http://dx.doi.org/10.1016/j.suc.2017.06.003

Recebido: 12 de janeiro de 2021

Aprovado: 27 de maio de 2021

Publicado: 22 de julho de 2021

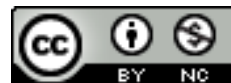

A Revista Baiana de Enfermagem utiliza a Licença Creative Commons - Atribuição-NãoComercial 4.0 Internacional. https://creativecommons.org/licenses/by-nc/4.0/ Este artigo é de acesso aberto distribuído sob os termos da Licença Creative Commons (CC BY-NC). Esta licença permite que outros remixem, adaptem e criem a partir do seu trabalho para fins não comerciais. Embora os novos trabalhos tenham de lhe atribuir o devido crédito e não possam ser usados para fins comerciais, os usuários não têm de licenciar esses trabalhos derivados sob os mesmos termos. 